\title{
Risk factors that can lead to development of meconium aspiration syndrome
}

\author{
Abstract \\ Objective: To determine the frequency of Meconium Aspiration Syndrome (MAS) in \\ neonates born through Meconium Stained Amniotic Fluid (MSAF) and to identify the \\ risk factors that can lead to the development of MAS.
}

Method: The study includes all neonates born in 2014 through MSAF. Risk factors associated with pregnancy, delivery and the neonates' condition were identified. Results between groups - the Meconium Aspiration Syndrome (MAS) group, the meconium in the lower vocal cords, but no Meconium Aspiration Syndrome (MLVC) group, and the MSAF group - were compared.

Results: Out of the 2040 births in 2014, 307 neonates were born through meconium stained amniotic fluid. MAS were confirmed in 17 neonates $(5.5 \%)$. Statistically significant differences between the MAS and the MSAF groups were found in the following: parity (MAS was more likely in nulliparous women (p-0.024, RR-1.8, 95\% CI 1.4-2.3; p-0.045), the BMI of women (28.2 \pm 4.1 in the MSAF group and $30.6 \pm 4.2$ in the MAS group (T-2.24; $\mathrm{p}-0.027)$ ), and respiratory tract infections and anaemia during pregnancy, which were more common in the MAS group ( $\mathrm{p}-0.02 ; \mathrm{p}-0.035)$. MAS was more likely to occur upon induction of labour, meconium was thick, and the duration of labour was longer in first and second stages (p-0.003, p- $0.0022, \mathrm{p}-0.022, \mathrm{p}-0.000)$. $60 \%$ of neonates who developed MAS were born through cesarean-section, compared to $31 \%$ born through cesarean-section in the MSAF group (p-0.043). Pathological changes in CTG were more common in the MAS group (47\% compared to $4 \%$ in the MSAF group, $\mathrm{p}-0.035$ ). The likelihood of MAS in neonates was found to increase with lower Apgar scores at minute 1 and minute 5; lower cord blood $\mathrm{pH}$ was also a risk factor for MAS (p-0.000, p-0.000, p-0.009).

Conclusions: Prolonged labour, lower Apgar scores, induction of labour, thick meconium, nulliparity, respiratory tract infections and anaemia during pregnancy were associated with MAS. Factors such as urinary tract infections, hypertensive disorders, neonate birth weight and sex were not found to be statistically significant in this study.
Volume 3 Issue 3 - 2018

\author{
Linas Rovas, ${ }^{1,2}$ Arturas Razbadauskas,' Emilija \\ Boguziene ${ }^{2}$ \\ 'Klaipeda University, Klaipeda, Lithuania \\ ${ }^{2}$ Woman and Child Clinic, Siauliai, Lithuania
}

Correspondence: Linas Rovas MD, Ph.D, Klaipeda University, Klaipeda,Women and Child Clinic, Architektu 77, LT-78I70 Siauliai, Lithuania, Tel +370-698-43875,

Email dr.linasrovas@gmail.com

Received: May 28, 2018 | Published: June 13, 2018

Keywords: meconium aspiration syndrome, newborn, cesarean section, pregnancy, apgar scores

Abbreviations: MAS, meconium aspiration syndrome; MSAF, meconium stained amniotic fluid; MLVC, meconium aspiration syndrome; GTIs, genital tract infections; UTIs, urinary tract infections; RTIs, respiratory tract infections; GBS, group B beta haemolytic streptococcus

\section{Introduction}

Meconium is a medical term, intended to describe the first stools of a newborn. Meconium appears in foetal intestines around 10-16 weeks into pregnancy. MSAF has been considered an indication of foetal compromise, and is associated with an increased risk for neonatal morbidity. ${ }^{1-4}$ MAS is a serious, life-threatening condition that occurs in around $2 \%$ to $5 \%$ of all births. ${ }^{1}$ MAS is defined as respiratory distress that develops shortly after birth with a history of meconium stained amniotic fluid and radiological evidence of aspiration pneumonitis. All neonates born through meconium stained amniotic fluid are at risk of developing MAS, which may lead to high mortality and morbidity. ${ }^{2,3}$ MSAF occurs in around $10-15 \%$ of labours. MSAF rarely occurs before the $30^{\text {th }}$ week of gestation. ${ }^{4}$ The incidence of this condition increases with longer gestation, with approximately $30 \%$ of newborns developing MSAF at 42 weeks. ${ }^{5}$ But why do some newborns born through MSAF develop MAS while others do not is not completely clear. Numerous studies have been carried out in order to identify possible risk factors of MAS. However, the study data are quite controversial. Risk factors confirmed by some studies have been refuted by others. Many studies have confirmed that thick meconium, low Apgar scores at minute 1 and minute 5, and non-reassuring FHR tracing were associated with MAS in MSAF pregnancies. ${ }^{6-9}$ In Sriram, et al., ${ }^{10}$ oligohydramnios and male gender had been suggested to increase the incidence of MAS. However, these findings were not confirmed by other studies. MAS should be largely preventable by careful antenatal monitoring, appropriate delivery room tactics for foetal distress and rapid resuscitation, although, despite all the efforts and regulations, MAS still happens. Consequently, knowing the risk factors is particularly important. The aim of this study was to identify the risk factors that can lead to MAS, including all possible risk factors from all possible groups. 


\section{Materials and methods}

A retrospective study was conducted, including all neonates born in 2014 through meconium stained amniotic fluid. The study includes women with at least 37 weeks of gestation. Women with multiple gestations, preterm deliveries and foetal deaths in utero were excluded from this study.

Risk factors were divided into 4 categories: a) maternal factors (age, education, parity, BMI, smoking and caesarean section in anamnesis); b) pregnancy-associated risk factors (hypertension, preeclampsia, eclampsia, iron-deficiency anaemia, AFI (Oli-, poly-), imminent preterm delivery, genital tract infections (GTIs), urinary tract infections (UTIs), respiratory tract infections (RTIs), Group B Beta Haemolytic Streptococcus (GBS); c) risk factors associated with labour (week of gestation, mode of delivery, labour induction, thickness of meconium, duration of the first labour stage, duration of the second labour stage, rupture of the membrane to delivery interval, dystocio, labour augmentation (use of oxytocin and prostaglandin E2), cardiotocograph trace during labour (normal/reassuring; nonreassuring; abnormal); d) risk factors associated with the newborn (sex of the newborn, weight of the newborn, Apgar score at minute 1 and minute 5, umbilical cord blood gas $\mathrm{pH}$, duration of hospitalisation, and final diagnosis of the newborn).

Relationships between maternal risk factors, pregnancy-associated risk factors, labour- associated risk factors and newborn risk factors were compared in three groups: the MAS group (MAS), the MLVC group and the MSAF group.

Differences between categorical variables were analysed using Pearson's chi-square test and Fisher's exact test. Differences between continuous variables were analysed using Tukey's range test and Student's t-test. The difference was considered statistically significant if $\mathrm{P}$ value was $<0.05$. Statistical analysis was performed using IBM SPSS Statistics, version 22.0.

\section{Results}

During the period between 01 January 2014 and 31 December 2014, 2040 babies were delivered in our clinic. 321 were born through MSAF, which constitutes $15.7 \%$ of all births. Excluding multiple gestation pregnancies, preterm deliveries and fetal deaths in utero, 307 cases were included in the final study. 34 neonates were found to have meconium in their lower vocal cords (MLVC) without developing Meconium Aspiration Syndrome. Meconium Aspiration Syndrome developed in 17 newborns. MAS frequency was $5.5 \%$ in all neonates born through MSAF. The mortality rate from MAS for neonates was $5.8 \%(1 / 17)$.

Maternal risk factors between the MAS, MLVC and MAS groups were analysed. MAS were found to be more likely in nulliparous women.

For nulliparous women, the probability of developing MAS is two times higher, as compared to multiparous women. (x2-5.1, p-0.024, RR-1.8 (95\% CI 1.4-2.3; p-0.045). The BMI of mothers in the MAS group was also found to be higher, as compared to mothers in the MSAF group (28.2 \pm 4.1 and $30.6 \pm 4.2 ; \mathrm{t}-2.24, \mathrm{p}-0.027)$ (Table 1). Another statistically significant difference between the MAS and the MSAF groups was found in smoking. Smoking increases the relative risk of MAS three-fold (RR-3.48; 95\% CI 2.17-5,58; p-0.027). However, a woman's age and education did not affect the occurrence of MAS. A history of caesarean sections has no influence on the likelihood of developing MAS either.

In the pregnancy-associated risk factors, statistically significant differences were found for the following factors: iron deficiency anaemia and respiratory tract infections (RTIs) during pregnancy.

Respiratory tract infections (RTIs) and anaemia during pregnancy were more common in the MAS group (p-0.02; $\mathrm{p}-0.035)$. RTIs during pregnancy increase the risk of MAS two-fold (p-0.012, RR-2.5 CI 1.5-4.2). Anaemia during pregnancy increases the risk of MAS 2.5 times (p-0.035, RR-2.45, 95\% CI-1.78-3.37). There was no difference in the frequency of gestational hypertension, pre-eclampsia and eclampsia between groups. There was also no difference in the frequency of genital tract infections (GTIs) and urinary tract infection (UTIs) between groups. Although not statistically significant, it is worth mentioning that BHS was diagnosed more often in the MAS group $(5.8 \%$ and $9.8 \%, p>0.05)$. The subjects in the MAS group were also more likely to develop imminent preterm pregnancy, although the difference was not statistically significant $(25 \%$ and $12 \%$ respectively, $\mathrm{p}>0.05$ ) (Figure 1).

Table I Differences between continuous variables

\begin{tabular}{|c|c|c|c|}
\hline Variable & MSAF group & MAS group & $\mathbf{p}$ \\
\hline Maternal age & $27,2 \pm 5,2(N-273)$ & $25,3 \pm 5,6(\mathrm{~N}-17)$ & 0,095 \\
\hline Maternal BMI & $28,2 \pm 4, I(N-273)$ & $30,6 \pm 4,2(N-17)$ & 0,027 \\
\hline I labor stage duration (vaginal deliveries only) & $6 \mathrm{val}+50 \mathrm{~min}(\mathrm{~N}-198)$ & $10 \mathrm{val}+10 \min (\mathrm{N}-7)$ & $\mathrm{p}-0,022$ \\
\hline II labor stage duration (vaginal deliveries only) & $22,79 \pm 15 \min (N-198)$ & $56,12 \pm 16 \min (N-7)$ & $\mathrm{p}-0,000$ \\
\hline Rupture membranes to birth duration (vaginal deliveries only) & $\begin{array}{l}4 \mathrm{hl} \text { min } \pm 3 \mathrm{~h} 47 \mathrm{~min} \\
(\mathrm{~N}-198)\end{array}$ & $\begin{array}{l}\text { 6h } 14 \min \pm 2 \mathrm{~h} 18 \mathrm{~min} \\
(\mathrm{~N}-7)\end{array}$ & $\mathrm{p}-0,025$ \\
\hline Newborn weight, g & $3540 \pm 509(N-273)$ & $3562 \pm 562(\mathrm{~N}-17)$ & $p-0,36$ \\
\hline Umbilical Cord pH & $7,34 \pm 0,08(\mathrm{~N}-144)$ & $7,27 \pm 0,12(\mathrm{~N}-17)$ & $p-0,009$ \\
\hline
\end{tabular}




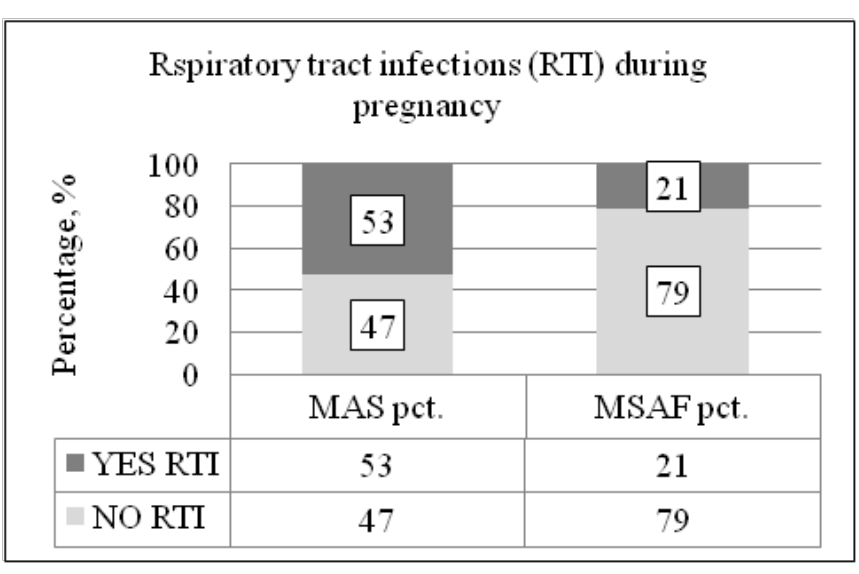

Figure I RTI during pregnancy.

An evaluation of delivery-related risk factors have shown the following to have an effect on the frequency of MAS: density of meconium, labour induction, duration of the first and second labour stages, rupture of the membrane to delivery interval and cardiotocograph trace changes during labour. If meconium is thick, the likelihood of MAS is increased 6.5 times (p-0.0022, RR- $6.595 \%$ CI 3.65 to 10.8). If labour is induced, the probability of MAS increases by almost 3.5 times (p-0.003, RR-95 3.4\% CI 1.89 to 6.1). Isolating only vaginal deliveries between the MAS and MSAF groups has shown that the first and second labour stages are longer in the MAS group. In addition, rupture of the membrane to delivery is also longer in the MAS group (Table 1). An assessment of the cardiotocograph trace changes between the groups has shown that non-reassuring and abnormal cardiotocography changes were more frequent in the MLVC and MAS groups, as compared to the MSAF group (Figure 2).

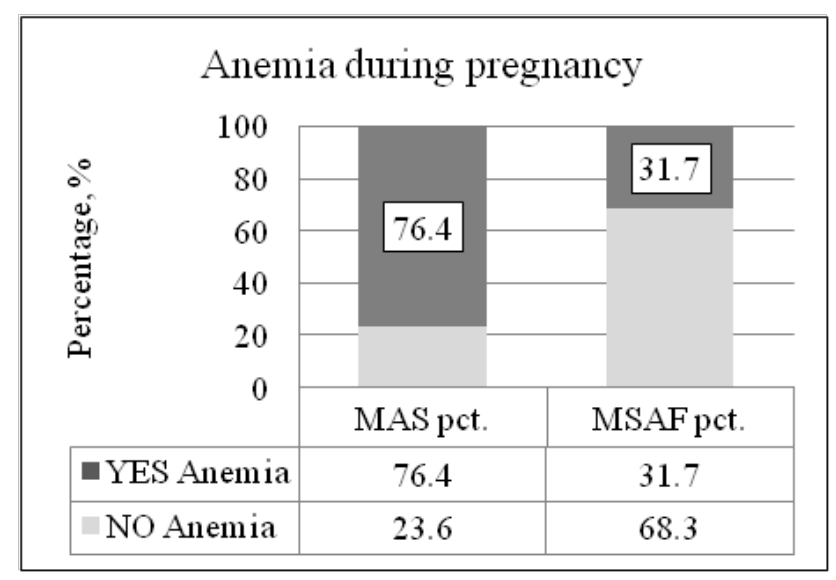

Figure 2 Anemia during pregnancy.

An interpretation of the data on the mode of delivery has shown that caesarean section is performed more frequently in the MAS group. In the MSAF group, $69 \%$ of newborns were born by vaginal delivery and $31 \%$ by cesarean-section; in the MLVC group, caesarean section was performed in $35 \%$ of cases; and in the MAS group, only $41 \%$ of newborns were born naturally, while $59 \%$ of women (i.e., 10 out of 17) gave birth by caesarean section (Figure 3).
Another finding was that the gestational age at delivery differed statistically significantly between the MSAF, MLVC and MAS group. In the MSAF and MLVC groups, labour at week 42 (week $41+0$ days, week $-41+6$ days) occurs or is induced in $24 \%$ of cases, as compared to $35 \%$ of cases in the MAS group.

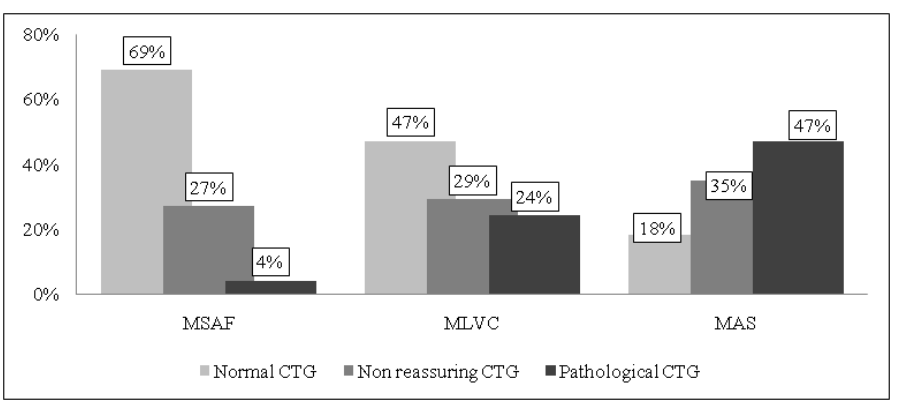

Figure 3 Cardiotocograph traces in different groups.

An analysis of the newborn risk factors has shown statistically significant differences between groups in the following factors: Apgar 1 and Apgar 5 cores, umbilical cord blood $\mathrm{pH}$, and the duration of hospitalisation. No statistically significant differences were found between the newborn's sex and weight. In the MSAF group, the Apgar 1 score is 7-9 in 80\% cases, as compared to an Apgar score of 6-7 in $70 \%$ of cases in the MAS group. In addition, Apgar 5 was lower (68 ) in the MAS group in $70 \%$ of cases, as compared to $8-10$ in $80 \%$ cases in the MSAF group. Umbilical cord $\mathrm{pH}$ was also lower in the MAS group $-7.27 \pm 0.12$, compared to $7.34 \pm 0.08$ in the MSAF group $(\mathrm{t}-2.64, \mathrm{p}-0.009)$. The average hospitalisation time for newborns delivered through stained amniotic fluid who had no meconium below the vocal cords was $2.5 \pm 2$ days, as compared $4,1 \pm 2,5$ days $(\mathrm{p}<0,05)$ in the MLVC group, or $6.9 \pm 3$ days $(\mathrm{p}<0,05)$ when MAS occurred.

\section{Discussion}

The present study has shown that all risk factor groups are important to the development of the meconium aspiration syndrome. Discussing maternal variables it is very important to highlight the significance of BMI. The significance of BMI in the development of MAS has also been described by Martina Persson, et al., ${ }^{11}$ The risk of meconium aspiration increases together with increases in maternal BMI. The present study had also found that nulliparity is also an important factor in developing MAS. Other studies had also established the significance of nulliparity as a variable for MAS. ${ }^{12-15}$ The present study had also shown that smoking increases the risk of meconium aspiration syndrome. While this variable is not widely discussed in research, Usta M, et al., ${ }^{7}$ had found a contradictory effect, showing that smoking was associated with a lower risk of meconium aspiration, while Benny PS had found the likelihood of meconium aspiration increased if the mother was a smoker (i).

Discussing the variables associated with pregnancy, we have not determined any statistically significant differences between groups with regards to hypertensive pregnancy disorders. Other studies have shown that pregnancy-associated hypertension, pre-eclampsia and eclampsia are related to increased risk for the meconium aspiration syndrome. ${ }^{16,17}$ On the other hand, the relation between hypertensive pregnancy disorders and meconium aspiration had not been found 
in many other studies. ${ }^{12,14,16,17}$ Therefore, on the basis of the present as well as other studies, we propose that hypertensive pregnancyassociated disorders are not strong risk factors for the meconium aspiration syndrome. Other pregnancy-associated conditions, such as genital and urogenital tract infections, were not important for the present study. Although not statistically significant, BHS colonisation was found to be higher in the MSAF group, as compared to the MAS group, which raises a hypothesis of the syndrome's protective antibiotic value and infectious origin. This idea is also supported by the higher rate of imminent pre-term pregnancies in the MAS group due to the large proportion of premature births being associated with infection. Unfortunately, the difference was statistically significant The present study had also found that respiratory tract infections during pregnancy, as well as anaemia, are associated with a higher risk of pregnancy. Anaemia as a risk factor for MAS was also confirmed by Benny PS et al. However, we have not found that respiratory tract infections during pregnancy have any impact on the development of MAS. For this reason, the statistically significant variable and Ghidini et al., ${ }^{18}$ suggest a number of interesting ideas related to meconium aspiration and its pathogenesis. This should lead us to at least consider the possibility that meconium aspiration is only one of several factors that lead to meconium aspiration syndrome.

Speaking of birth itself, the tactics used during delivery play an important role in the appearance of MAS. Prolonged birth and prolonged ruptured membranes to birth time are associated with meconium aspiration syndrome. Similar results have been demonstrated by Benny PS et al., ${ }^{19}$ where meconium aspiration was associated with prolonged labour. Interesting results can also be found in Woneui C. et al., ${ }^{20}$ where severe MAS was associated with a shorter duration of the second stage of labour, and mild to moderate meconium aspiration syndrome - with prolonged labour. This suggests that birth through meconium stained amniotic fluid should not be prolonged and, if other warning signs are manifest during labour, should be terminated in the safest and quickest way possible. In congruence with past research, the present study had also confirmed that thick meconium, labour induction and non-reassuring or abnormal CTG are associated with a massive increase in risk of meconium aspiration. ${ }^{6-22}$ This means that, once labour is induced and thick meconium present, it should only be continued if CTG is normal. If CTG is abnormal or non-reassuring and meconium is thick, there is no need to be afraid of making tough decisions. Like in all above mentioned studies, newborn variables were also very important. The present study had also confirmed that lower Apgar scores and lower umbilical cord blood $\mathrm{pH}$ values are associated with meconium aspiration syndrome. ${ }^{2-18}$ This means that even in cases where no warning signs are present during birth or pregnancy, newborns born through MSAF with lower Apgar scores at minutes 1 and 5 are at a higher risk of developing MAS, and should therefore be inspected more carefully. ${ }^{23-29}$

\section{Conclusion}

The present study had found many of the same phenomena as a number of other studies, as well as a few interesting factors related to the development of the meconium aspiration syndrome. We wanted to show that all factors groups-maternal, pregnancy, labour and newborn-are important and should be evaluated very carefully, because all groups were found to contain factors that bear on the development of the meconium aspiration syndrome. Finally, we would like to add that there are still many aspects of meconium aspiration, which sadly is a frequent cause of newborn morbidity and mortality that are yet to be discovered.

\section{Acknowledgments}

None.

\section{Conflicts of interest}

The author declares that they do not have any conflicts of interest.

\section{References}

1. Singh BS, Clark RH, Powers RJ, et al. Meconium aspiration syndrome remains a significant problem in the NICU: outcomes and treatment patterns in term neonates admitted for intensive care during a ten-year period. J Perinatol. 2009;29(7):497-503.

2. Ziadeh SM, Sunna E. Obstetric and perinatal outcome of pregnancies with term labor and meconium stained amniotic fluid. Arch Gynecol Obstet. 2000;264(2):84-7.

3. Beligere N, Rao R. Neurodevelopmental outcome of infants with meconium aspiration syndrome: report of a study and literature review. $J$ Perinatol. 2008;28(Suppl 3):S93-101.

4. Meconium staining of the amniotic fluid (MSAF) occurs in around 10$15 \%$ of labours. MSAF rarely occurs before 30 weeks gestation.

5. Wiswell TE. Handling the meconium-stained infant. Semin Neonatol. 2001;6(3):225-31.

6. Khazardoost S, Hantoushzadeh S, Khooshideh M, et al. Risk factors for meconium aspiration in meconium stained amniotic fluid. $J$ Obste Gynaecol. 2007;27(6):577-9.

7. Usta IM, Mercer BM, Sibai BM. Risk factors for meconium aspiration syndrome. Obstet Gynecol. 1995;86(2):230-4.

8. Urbaniak KJ, McCowan LM, Townend KM. Risk factors for meconiumaspiration syndrome. Aust N Z J Obstet Gynaecol. 1996;36(4):401-6.

9. Meydanli MM, Dilbaz B, Calişkan E, et al. Risk factors for meconium aspiration syndrome in infants born through thick meconium. Int $J$ Gynaecol Obstet. 2001;72(1):9-15.

10. Sriram W, Khoshnood B, Singh JK, et al. Racial disparity in meconium stained amniotic fluid and MAS in the US, 1989-2000. Obstet Gynecol. 2003;102(6):1262-8.

11. Persson M, Johansson S, Villamor E, et al. Maternal Overweight and Obesity and Risks of Severe Birth-Asphyxia-Related Complications in Term Infants: A Population-Based Cohort Study in Sweden. PLoS Med. 2014;11(5):e1001648

12. Meis PJ, Hall III M, Marshall JR, et a. Meconium passage: a new classification for risk assessment during labor. Am J Obstet Gynecol. 1987;131(5):509-13.

13. Falciglia HS, Henderschott C, Potter P, et al. Does DeLee suction at the perineum prevent meconium aspiration syndrome? Am J Obstet Gynecol. 1992;167(5):1243-9.

14. Alexander GR, Hulsey TC, Robillard PY, et al. Determinants of meconium stained amniotic fluid in term pregnancies. J Perinatol. 1994;14(4):259 63

15. Meydanli MM, Dilbaz B, Calișan E, et al. Risk factors for meconium aspiration syndrome in infants born through thick meconium. Int $J$ Gynaecol Obstet. 2001;72(1):9-15. 
16. Dargaville PA, Copnell B. The epidemiology of meconium aspiration syndrome: incidence, risk factors, therapies, and outcome. Pediatrics. 2006;117(5):1712-21.

17. Paz Y, Solt I, Zimmer EZ. Variables associated with meconium aspiration syndrome in labours with thick meconium. Eur J Obstet Gynecol Reprod Biol. 2001;94(1):27-30.

18. Ghidini A, Spong CY. Severe meconium aspiration syndrome is not caused by aspiration of meconium. Am J Obstet Gynecol. 2001;185(4):931-8.

19. Benny PS, Malani S, Hoby MA, et al. Meconium aspiration--role of obstetric factors and suction. Aust NZJObstet Gynaecol. 1987;27(1):36-9.

20. Choi W, Jeong $\mathrm{H}$, Choi SJ, et al. Risk factors differentiating mild/ moderate from severe meconium aspiration syndrome in meconiumstained neonates. Obstet Gynecol Sci. 2015;58(1):24-31.

21. Anwar Z, Butt TK, Kazi MY. Mortality in meconium aspiration syndrome in hospitalized babies. J Coll Physicians Surg Pak. 2011;21(11):695-9.

22. Vivian-Taylor J, Sheng J, Hadfield RM, et al. Trends in obstetric practices and meconium aspiration syndrome: a population-based study. BJOG. 2011;118(13):1601-7.
23. Eden RD, Seifert LS, Winegar A, et al. Perinatal characteristics of uncomplicated postdate pregnancies. Obstet Gynecol. 1987;69:(3 Pt 1):96-299.

24. Krebs HB, Petres RE, Dunn LT, et al. Intrapartum Fetal Heart Rate Monitoring. III. Association of meconium with abnormal fetal heart rate patterns. Am J Obstet Gynecol. 1980;137(8):936-43.

25. Fanaroff AA. Meconium aspiration syndrome: historical aspects. $J$ Perinatol. 2008;28(Suppl 3):S3-7.

26. Ross MG. Meconium aspiration syndrome: more than intrapartum meconium. N Engl J Med. 2005;353(9):946-8.

27. Karatekin G, Kesim MD, Nuhoglu A. Risk factors for meconium aspiration syndrome. Int J Gynaecol Obstet. 1999;65(3):295-7.

28. Bhutani VK. Developing a systems approach to prevent meconium aspiration syndrome: lessons learned from multinational studies. $J$ Perinatol. 2008;28(Suppl 3):S30-35.

29. Yoder BA, Kirsch EA, Barth WH, et al. Changing obstetric practices associated with decreasing incidence of meconium aspiration syndrome. Obstet Gynecol. 2002;99(5 Pt 1):731-9. 